\title{
Visibility in the digital age: Introduction
}

\author{
Cornelia Brantner*, Karlstad University, Department of Geography, Media and Communication, \\ Sweden \\ Helena Stehle, University of Münster, Department of Communication, Germany \\ * Corresponding author: cornelia.brantner@kau.se
}

In the digital age, calls for transparency and openness as well as for privacy and confidentiality prevail: Struggles for visibility occur simultaneously with conflicts regarding invisibility and hidden battles for power and privileges of interpretation. Concerns about a loss of digital self-determination exist, just like those regarding the "right to be forgotten" or the right to become invisible and unseen. While the idea of a "transparent user" - as the ultimate notion of (in)voluntary visibility - has caused a broad outcry in society and in scientific debates a few years ago (Palfrey \& Gasser, 2008), the discussion has shifted toward considerations of Internet governance and regulation (Camenisch, Fischer-Hübner, \& Hansen, 2015). Brighenti (2010, p. 109) has pointed out that visibility has long been one of the key aspects "associated with the public sphere" and that in today's digitized publics, the "project of democracy can no longer be imagined without taking into account visibility and its outcomes" (Brighenti, 2010, p. 189). Visibility and invisibility, along with their societal outcomes, are increasingly being discussed and analyzed, as they are becoming important dimensions in the accurate description and explanation of digital communication. They can be addressed with regard to individuals and institutions (e.g., their ability to speak, their power, or opinion leadership, etc.), structures and processes (e.g., in terms of becoming visible or making visible), and data and information (e.g., their accessibility and comprehensibility, or their exploitation).

While the original meaning of visibility is closely related to spatiotemporal proximity and the human senses, especially the visual sense, this link is effectively dissolved in the digital space (Thompson,
2005). "Digital visibility" refers to perceptibility as the likelihood of being 'seen' in the sense of being noticeable (this understanding is closest to the original understanding), in being heard or noticed, or in the sense of being respected or recognized. This distinction points to at least three different levels of visibility, which have been discussed in varying degrees in different fields of communication and media studies.

The first level refers to what Leonardi and Treem (2020, p. 12, emphasis added by the authors) define as "the sociomaterial performance of the behavior of people, collectives, technological devices, or nature in a format that can be observed by third parties through minimal effort". It is also evident, for example, when studies look at whether and how actors (e.g., journalists, scientists, politicians, teachers, media outlets, corporations, political institutions, etc.) seek - intentionally or unintentionally - visibility or invisibility or become potentially recognizable (e.g., Bock \& Probst, 2018; Cruz, 2017; Flyverbom, Leonardi, Stohl, \& Stohl, 2016; Karlsson, 2011; Wilhelm, Stehle, \& Detel, 2021). Stohl, Stohl, and Leonardi (2016, p. 125) shed light on actors' (strategic) handling of visibility by analyzing it as "(1) the availability of information, (2) approval to share information, and (3) the accessibility of information to third parties".

The first level is also often linked to "visibility as presence" in traditional media, which refers to "being noticed" or "being heard" by the media (e.g., Bantimaroudis, Zyglidopoulos, \& Symeou, 2010). Likewise, media presence itself reveals connections to the second level: For example, opportunities for the second level ("being heard" by others) open up when 
someone or something is present in the media. Research on media content and media use can also be situated at the interface between the first and second levels, for example, regarding media salience, awareness, attention, or prominence of an object or content (e.g., Kiousis, 2004).

The third level is often discussed in research on diversity or inequality, which focuses on "being recognized" or "being respected". This research addresses structures, conditions, and constraints of equality or inequality, especially with regard to gender issues or diversity. Dahlberg (2018) associates visibility with conceptions of the public sphere for which mutual recognition and participatory equality (among others) are normative requirements. Meanwhile, Brighenti (2007) points to the close association of visibility to social recognition and to visibility as empowerment. Furthermore, the author defines recognition as one of two possible outcomes of visibility, with the other being control.

These examples demonstrate that the three levels of digital visibility are by no means unrelated. Rather, they are often closely linked to one another and may even merge to a certain extent. Accordingly, defining visibility is not straightforward; and the conception is to some extent one of emphasis and nomenclature. Furthermore, the concept of "visibility" is linked to other concepts, such as "transparency" (Stohl et al., 2016) or "legitimacy" (Koopmans, 2004). In his work on social movements and the recognition of their causes and aims by the "mainstream" public sphere, Koopmans (2004) defines visibility as a precondition of legitimacy. In such a definition, visibility is located at the second level, among the three levels mentioned. However, other authors, such as Brighenti (2007) and Dahlberg (2018), define legitimacy or recognition as a form of visibility. As expected, research has indeed shown that visibility at the first two levels does not necessarily lead to visibility at the third level (e.g., Brantner, Lobinger, \& Stehling, 2020).

In summary, the different concepts focus on visibility and/or invisibility in var- ious ways with respect to the three levels. These also incorporate the characteristics of digital communication in different ways (e.g., with regard to objects, agents, or technological contexts of digital (in) visibility). All these aspects, when combined, make it difficult to take a systematic look at the field. This is exacerbated by the fact that related studies are scattered across various research fields in communication and media studies. Therefore, this Thematic Section aims to present cutting-edge research on visibility and invisibility in digital communication, especially those that take up the three levels of digital visibility in various forms.

The articles gathered in this Thematic Section contribute to the burgeoning field of visibility research in communication and media studies. These works shall provide the ground for further discussions and studies on the social, cultural, and political conditions, patterns, and implications of online visibility and invisibility. They encompass both classical and new fields of research regarding (in)visibility, such as research on inequality, diversity, and representation, as well as research on science communication and location-based apps. Three authors take the more theoretical and/or conceptual approach by reviewing the literature on their specific topics: Claudia Wilhelm on gendered (in)visibility, Darryl A. Pieber on locative media and social accessibility, and Julia Metag on the tension between visibility and invisibility in science communication. Three authors use empirical data to reveal new insights into (in)visibility in digital communication contexts, namely, Christine Linke and Elizabeth Prommer on the diversity of media representation and production culture, and Dafne Calvo on inequalities in online communities in Spain.

Claudia Wilhelm strives to characterize gendered digital visibility based on a literature review of gender and feminist research as well as of communication and media studies. She focuses on women's participation in digital communication (especially on social media), on the role gender plays in digital media environments, and on aspects that drive or inhibit 
women's digital visibility. Wilhelm identifies the representation and integration of all genders, gender norms, and power relations as sub-dimensions of digital visibility, thus primarily addressing the third visibility level. She particularly draws upon the example of the \#MeToo movement and proposes a taxonomy of research based on the patterns of replication, reinforcement, and resilience. These patterns show how the role played by gender in digital media environments is framed in the literature reviewed. By replication, Wilhelm refers to the process of analyzing digital visibility against the background of (non-diverse) binary gender conceptions from the offline world, such as regarding the integration of women in discussions, while the reinforcement pattern points to characteristics of digital media environments that amplify gendered inequalities (e.g., anonymity, invisibility, etc.). The literature assigned to the resilience pattern, meanwhile, addresses empowerment and aspects that foster the visibility and inclusion of all genders in digital media environments. Thus, Wilhelm shows how the literature deals with the digital visibility of women and how the interplay between technological aspects and social norms can be explored.

In his paper on locative media apps, Darryl Pieber links visibility to Simmel's (1992, 1955) concept of "social accessibility". The author states that social accessibility, defined as the ability to be reached by others, is only possible when a person is visible. Thus, he first addresses the first visibility level but later extends it to the third level. Pieber reviews and synthesizes the existing literature, which regards the ways in which people use location-based media apps to manage their social accessibility and, therefore, their visibility in the urban environment. Using a narrow definition, Pieber further focuses on location-based apps that utilize the location coordinates of users' mobile devices and present them with information about their surroundings. The author points out that while these apps are being increasingly used, the non-use by certain groups, the biases embedded in the algorithms, or the unwillingness of people to squeeze themselves into categories set by the app lead to the invisibility of certain people and groups. These aspects can even reinforce racial and social divides by preventing people from developing what is called an "indifference to difference" (Tonkiss, 2005) in urban spaces. However, both visibility and invisibility within these apps are associated with certain downsides. While being invisible entails the risk of being further marginalized, being visible also carries the risks of potential surveillance. Pieber concludes that the existing studies show that location-based apps have a tendency to oversimplify or filter out differences among their urban users - processes in which existing manifestations of racism and prejudice are reinforced and solidified. Following Young (1986), Pieber argues that making the complexity of differences invisible is "anti-urban".

Scientific findings, along with the people behind them, are being increasingly placed under the watchful public eye or are being asked to be made publicly visible. In her work, Julia Metag addresses and theoretically models the tensions that accompany science communication in digital contexts. She demonstrates how science communication research focuses on public visibility, which is particularly understood as media presence (and thus primarily addressing the second visibility level), but often forgets to consider tensions and divergences that accompany it (e.g., between journalistic and scientific logic). In her analysis, the author first makes a distinction between the visibility of scientific knowledge and that of scientists as actors. Then, she further distinguishes between two reference systems of such visibility: that of the science system itself and that of the public. In doing so, she shows how different tensions arise depending on visibility and frame of reference. Based on a discussion of key paradigms of science communication research, Metag concludes her work by addressing three concerns: the consequences of visibility in digital media environments for scientists and scientific knowledge, the process in which visibility can be strategically man- 
aged, and where the limits of strategic visibility management might lie.

Christine Linke's and Elizabeth Prommer's contribution is one of the two empirical analyses of visibility in the Thematic Section. Like the contributions of Wilhelm, Pieber, and Calvo, Linke and Prommer address the visibility of difference as they discuss and analyze the visibility of diverse people in German audio-visual programs, with a focus on gender portrayals in fictional and non-fictional German TV productions. In their work, the authors explore visibility before and behind the screen, scrutinizing both the media production level and the media product level, and are concerned with representational visibility, thus addressing the first and second visibility levels, respectively. They base their study on gender theory and intersectionality theory and conceptualize visibility as an intersectionality dimension. The authors' perspective combines the normative goal of achieving equality with the deconstructivist interpretation of their findings against the background of societal and media power structures, culminating in an attempt to uncover the "doing gender" in media. Empirically, they apply content analysis to measure diversity in terms of the visibility of people of different genders, ages, professions, and roles on and behind the screen. In line with the assumptions, they find that women, and increasingly so with age, are less often visible on German television than men, both on and behind the screen. Other genders are invisible. Whether in fictional or nonfictional programming, women are less visible on television in almost all depicted occupations than their proportion in "real life" would suggest. While the study also reveals that the visibility of women at the production level is accompanied by increasing visibility of women on screen, they still comprise a low share of the total media production workforce. An additional analysis of online content reveals a similar picture in the German YouTube universe, wherein women are also not equally visible and are mainly shown in stereotypical gender roles.
Dafne Calvo's contribution completes the Thematic Section. The author examines the diversity of Spanish free software and free culture communities. From the perspective of intersectionality theory, Calvo defines visibility as the ability to discuss underrepresentation in these communities and thus addresses primarily the third visibility level. She seeks to identify visibility gaps in these communities and understand the communities' perspectives on visibility issues, such as whether and how they themselves identify and interpret visibility issues and develop tactics to deal with them. For this purpose, Calvo applies a multi-method design that combines an online questionnaire answered by 290 different communities and interviews with members of 37 of those communities. One result is that even though men and people with high educational levels and technical expertise are overrepresented, the proportion of women in the groups is relatively high compared to previous studies. This result, however, cannot hide the fact that the world of free software and free culture communities is predominantly a world inhabited by males. The author concludes that, on the one hand, there exists a tension between the ideal of community members' invisibility (in the sense of anonymity) and their willingness to recognize inequalities within the communities. On the other hand, in the interviews, community members reflected on the exclusion of certain segments of society. They also showed an awareness of the intersectionality of different categories of difference, in whose interplay the non-participation of certain segments of society in technological production may be grounded. Calvo concludes that, despite the underrepresentation of certain groups, inequalities are an issue of visibility that can be linked to the communities' goals of social justice and social change.

The articles of the Thematic Section discuss digital visibility from different angles and analyze it against the background of various fields of communication and media studies. These articles address the three levels of digital visibility in different ways and contextualize them with regard to 
the respective research fields. In each case, innovative perspectives on and approaches to open questions in the research fields are presented. First, Wilhelm systematizes a field of research based on the concept of digital visibility. Second, Pieber combines a classic theory with an analysis of modern technology and its opportunities and challenges. Third, Metag takes a look at a hitherto rarely examined issue within science communication, whereas Linke and Prommer address multimodal media realities with an innovative empirical method. Finally, Calvo uses a multi-method design to gain insights into communities that value their anonymity.

As shown by both the introduction and the contributions to the Thematic Section, when it comes to digital communication, digital (in)visibility is a fruitful analytical concept for conducting research in communication and media studies. With regard to the three levels of digital visibility (being noticeable, being heard, and being recognized), intriguing questions remain open in relation to the manifold perspectives on this subject and the particularities of digital communication that have not yet been conclusively grasped (e.g., multimodality, blending of human and technical processes of perception and selection). They include questions about the transferability of findings about digital visibility to digital invisibility and of findings about the legacy media world to the digital world, as well as questions about the significance of technical aspects for digital (in)visibility and the blending of human and machine.

Last but not least, ongoing discussions on the advantages and disadvantages of both (un)intended visibility and (un)intended invisibility (e.g., in the context of surveillance and digital hate, on the one hand, and participation in discourse and empowerment on the other) must be continued. This Thematic Section hopes to stimulate further exchanges on these and other issues related to intentional and unintentional digital (in)visibility.

\section{References}

Bantimaroudis, P., Zyglidopoulos, S., \& Symeou, P. C. (2010). Greek museum media visibility and museum visitation: An exploration of cultural agenda setting. Journal of Communication, 60(4), 743-758. https://doi. org/10.1111/j.1460-2466.2010.01512.x

Bock, A., \& Probst, L. (2018). Opening up the classroom: Enabling and interrupting digital media practices in school. Education in the North, 25(3), 130-138. https://doi. org/10.26203/0cqq-5f74

Brantner, C., Lobinger, K., \& Stehling, M. (2020). Memes against sexism? A multi-method analysis of the feminist protest hashtag \#distractinglysexy and its resonance in the mainstream news media. Convergence, 26(3), 674-696. https://doi. org/10.1177/1354856519827804

Brighenti, A. (2007). Visibility: A category for the social sciences. Current Sociology, 55(3), 323-342. https://doi. org/10.1177/0011392107076079

Brighenti, A. M. (2010). Visibility in social theory and social research. London: Palgrave Macmillan. https://doi. org/10.1057/9780230282056

Camenisch, J., Fischer-Hübner, S., \& Hansen, M. (Eds.). (2015). Privacy and identity management for the future internet in the age of globalisation. Cham: Springer International. https://doi.org/10.1007/978-3319-18621-4

Cruz, J. M. (2017). Invisibility and visibility in alternative organizing: A communicative and cultural model. Management Communication Quarterly, 31(4), 614-639. https:// doi.org/10.1177/0893318917725202

Dahlberg, L. (2018). Visibility and the public sphere: A normative conceptualisation. Javnost - The Public, 25(1-2), 35-42. https://doi.org/10.1080/13183222.2018.1 418818

Flyverbom, M., Leonardi, P., Stohl, C., \& Stohl, M. (2016). The management of visibilities in the digital age: Introduction. International Journal of Communication, 10, 98-109. Retrieved from https://ijoc.org/ index.php/ijoc/article/view/4841

Karlsson, M. (2011). The immediacy of online news, the visibility of journalistic processes and a restructuring of journalistic au- 
thority. Journalism, 12(3), 279-295. https:// doi.org/10.1177/1464884910388223

Kiousis, S. (2004). Explicating media salience: A factor analysis of the New York Times issue coverage during the 2000 U.S. presidential election. Journal of Communication, 54(1), 71-87. https://doi. org/10.1111/j.1460-2466.2004.tb02614.x

Koopmans, R. (2004). Movements and media: Selection processes and evolutionary dynamics in the public sphere. Theory and Society, 33(3/4), 367-391. https://doi. org/10.1023/B:RYSO.0000038603.34963.de

Leonardi, P., \& Treem, J. W. (2020). Behavioral visibility: A new paradigm for organization studies in the age of digitization, digitalization, and datafication. Organization Studies, 41(12), 1601-1625. https://doi. org/10.1177/0170840620970728

Palfrey, J. G., \& Gasser, U. (2008). Born digital: Understanding the first generation of digital natives. New York: Basic Books.

Simmel, G. (1922 [1955]). The web of group affiliations (R. Bendix, Trans.). In G. Simmel (Ed.), Conflict and the web of group affiliations (pp. 125-195). Glencoe: Free Press.
Stohl, C., Stohl, M., \& Leonardi, P. M. (2016). Managing opacity: Information visibility and the paradox of transparency in the digital age. International Journal of Communication, 10, 123-137. Retrieved from https://ijoc.org/index.php/ijoc/article/ view/4466

Thompson, J. B. (2005). The new visibility. Theory, Culture \& Society, 22(6), 31-51. https:// doi.org/10.1177/0263276405059413

Tonkiss, F. (2005). Space, the city and social theory: Social relations and urban forms. Cambridge: Polity.

Wilhelm, C., Stehle, H., \& Detel, H. (2021). Digital visibility and the role of mutual interaction expectations: Reframing the journalist-audience relationship through the lense of interpersonal communication. New Media \& Society, 23(5), 1004-1021. https://doi. org/10.1177/1461444820907023

Young, I. M. (1986). The ideal of community and the politics of difference. Social Theory and Practice, 12(1), 1-26. Retrieved from https://www.jstor.org/stable/23556621 